\title{
Insertional mutations exhibiting high cell-culture density HCD phenotypes are enriched through continuous subcultures in Chlamydomonas reinhardtii
}

\author{
Leena Thung ${ }^{1}$, Jing $\mathrm{He}^{2}$, Qingling $\mathrm{Zhu}^{3}$, Zhenyu $\mathrm{Xu}^{3}$, Jianhua $\mathrm{Liu}^{2,3, *}$ and Yvonne Chow ${ }^{1}$ \\ ${ }^{1}$ Institute of Chemical Engineering and Sciences, A-STAR, Singapore 627833, Singapore \\ ${ }^{2}$ Ocean Research Centre of Zhoushan, Zhejiang University, Zhoushan 316021, China \\ ${ }^{3}$ Ocean College, Zhejiang University, Zhoushan 316000, China
}

Low efficiency in microalgal biomass production was largely attributed to the low density of algal cell cultures. Though mutations that reduced the level of chlorophyll or pigment content increased efficiency of photon usage and thus the cell-culture density under high-illumination growth conditions (e.g., $>500 \mu \mathrm{mol}$ photon $\mathrm{m}^{-2} \mathrm{~s}^{-1}$ ), it was unclear whether algae could increase cell-culture density under low-illumination conditions (e.g., $\sim 50 \mu \mathrm{mol}$ photon $\mathrm{m}^{-2} \mathrm{~s}^{-1}$ ). To address this question, we performed forward genetic screening in Chlamydomonas reinhardtii. A pool of $>1,000$ insertional mutants was constructed and subjected to continuous subcultures in shaking flasks under low-illumination conditions. Complexity of restriction fragment length polymorphism (RFLP) pattern in cultures indicated the degree of heterogeneity of mutant populations. We showed that the levels of RFLP complexity decreased when cycles of subculture increased, suggesting that cultures were gradually populated by high cell-culture density (HCD) strains. Analysis of the 3 isolated HCD mutants after 30 cycles of subcultures confirmed that their maximal biomass production was $50-100 \%$ higher than that of wild type under low-illumination. Furthermore, levels of chlorophyll content in HCD mutant strains were similar to that of wild type. Inverse polymerase chain reaction analysis identified the locus of insertion in two of three HCD strains. Molecular and transcriptomic analyses suggested that two HCD mutants were a result of the gain-of-function phenotype, both linking to the abnormality of mitochondrial functions. Taken together, our results demonstrate that HCD strains can be obtained through continuous subcultures under low illumination conditions.

Key Words: continuous subcultures; green microalgae; high cell-culture density (HCD); insertional mutation; inverse-PCR

\section{INTRODUCTION}

The green microalga Chlamydomonas reinhardtii is a widely used unicellular model organism for the study of flagellar structure and function, genetics of basal bodies, chloroplast biogenesis, photosynthesis, light perception, cell-cell recognition, and cell cycle control (Harris 2001). Analysis of the genome of $C$. reinhardtii reveals the evo- lution of key animal and plant functions (Merchant et al. 2007). It is also used as a model for the study of biofuel production (Wang et al. 2009, Moellering and Benning 2010, Work et al. 2010).

Insertional mutagenesis is a popular methodology for screen and isolation of mutant strains with specific
(9) \$ This is an Open Access article distributed under the terms of the Creative Commons Attribution Non-Commercial License (http://creativecommons.org/licenses/by-nc/3.0/) which permits unrestricted non-commercial use, distribution, and reproduction in any medium, provided the original work is properly cited.
Received August 30, 2017, Accepted February 28, 2018

*Corresponding Author

E-mail: liujh2013@zju.edu.cn

Tel: +86-580-2186223, Fax: +86-580-2186317 
phenotypes in C. reinhardtii. This is because the known insert-DNA sequences facilitate the identification of the insertion site in isolated mutant strains. A number of methods such as plasmid rescue (Tam and Lefebvre 1993), inverse polymerase chain reaction (inverse-PCR) (Yoshioka et al. 2004), and thermal asymmetric interlaced polymerase chain reaction (Pollock et al. 2003) are developed for determination of genomic sequences flanking to known insert-DNA fragments. Inserts that contain antibiotic resistant marker genes such as AphVIII (Sizova et al. 2001) and Ble (Lumbreras et al. 1998) against paramomycin and bleomycin (respectively) are frequently adopted for mutagenesis in wild type cells. To ensure antibiotic resistance, marker genes are often driven by robust promoters such as RBCS promoter or PSAD promoter (Lumbreras et al. 1998, Sizova et al. 2001). In most cases, insertional mutagenesis creates loss-of-function mutations or null mutations.

Sub-saturation screening using insertional mutations for genes involved in photosynthesis, nitrate assimilation, and acclimation to sulfur deprivation have provided tremendous insight into mechanisms regulating these processes and made resources available to the study of respective processes in C. reinhardtii (Dent et al. 2005, González-Ballester et al. 2005, Pollock et al. 2005). The amenability of the C. reinhardtii genome makes it suitable as green cell-factories to produce value-added metabolites and heterologous proteins (León-Bañares et al. 2004). However, the biomass yield of $C$. reinhardtii is relatively low when compared to some industrial green microalgal strains. To improve the biomass productivity, insertional mutant strains with reduced pigment or truncated antenna are found to show increased biomass yield under high but not low illumination conditions (Polle et al. 2003). This is because truncated antenna cells on surface of photobioreactors shield less light so that cells under the surface receive more light than wild type cells under high illumination conditions (Polle et al. 2003). On the other hand, biomass yield of truncated antenna cells is similar to that of wild type because no significant amount of lights is shielded by cells on surface (Polle et al. 2003).

To explore the possibility for having mutants exhibiting high biomass yield under low-illumination conditions in C. reinhardtii, we performed forward genetic screening for insertional mutant strains exhibiting high cell-density (HCD) phenotypes from a pool of $>1,000$ individual insertional mutant strains as a proof-of-principal study. Through 30 cycles of continuous subcultures, we have isolated 3 non-allelic strains exhibiting HCD phenotypes under low-illumination growth conditions. HCD strains show no apparent decrease of chlorophyll content. Inverse-PCR analysis identified the insertion site in 2 out of 3 HCD mutants, namely the HCD1 and HCD3. In both cases, genes associated with mitochondrial function at the insertion sites are found to be transcriptionally induced. Together, our results show that HCD strains can be enriched through continuous subcultures under lowilluminations. During the preparation of this manuscript, Cheng et al. (2017) have published a large-scale screening of 150 thousand insertional mutants. This provides the great resources for near-saturation screening of HCD mutant cells using the continuous subculture methodology in the future. We propose that near-saturation screening against the 150 thousand insertional mutants using this continuous subculture methodology will allow gaining insight into molecular mechanisms for regulation of cell-culture density in C. reinhardtii and possibly other green algae.

\section{MATERIALS AND METHODS}

\section{Strains, plasmids, and culture growth conditions}

The C. reinhardtii strain CC503 and plasmid pSP124S are acquired from the Chlamy center (http://www.chlamy.org). CC503 is cultivated in high salt (HS) (Sueoka 1960) and TAP media (Harris 1989) under autotrophic and heterotrophic growth conditions (respectively) of illumination at $50 \mu \mathrm{mol}$ photon $\mathrm{m}^{-2} \mathrm{~s}^{-1}$, shaking at 70-90 rpm, temperature at $25^{\circ} \mathrm{C}$, and bubbling with $2-3 \% \mathrm{CO}_{2}$ at $\sim 100 \mathrm{~mL} \mathrm{~min}^{-1}$ as indicated when appropriate. Cell density is determined by colorimetric method (optical density [OD] at $750 \mathrm{~nm}$ wavelength) or gravimetric method (cell dry weight). Continuous subcultures of stationary-phase cells were carried out by taking culture ( 3 days, $~ 1.4$ OD) as inoculum to seed the fresh medium (0 day, $\sim 0.15$ OD) and continue cycles of subcultures.

\section{Analysis of chlorophyll contents}

To determine chlorophyll content, cells from $2 \mathrm{~mL}$ culture were harvested by centrifugation. The resulting cells were resuspended in $96 \%$ ethanol and broken in the glass-bead beater FastPrep homogenizer (MP Biomedicals, Solon, OH, USA). After incubation with ethanol for $2 \mathrm{~h}$ at $4^{\circ} \mathrm{C}$, cell debris was cleared by centrifugation. The resulting supernatant was subjected to OD measurement at the wavelength of 645 and $663 \mathrm{~nm}$, with the $96 \%$ etha- 
nol as blank. Total chlorophyll content (i.e., $\operatorname{chl} a$ and $b$ ) was estimated using a formula $\mathrm{C}\left(\mathrm{mg} \mathrm{L}^{-1}\right)=20.2 \mathrm{OD}_{645}+$ $8.05 \mathrm{OD}_{663}$ (Sartory and Grobbelaar 1984) and was converted to percent of cell dry weight.

\section{Analysis of cell diameters and numbers}

Cell diameters and numbers were determined by using a particle analyzer (Multisizer 3; Beckman Coulter Life Sciences, Indianapolis, IN, USA) following the manufacturer's instruction. In brief, cells were collected from fresh culture by centrifugation at 2,500 rpm, washed with phosphate buffered saline (PBS) and resuspended with PBS to the initial volume. PBS washed cells were mixed with electrolyte (Beckman Coulter Life Sciences) with a ratio of $1: 19 \mathrm{v} / \mathrm{v}$. Cells in electrolyte were subjected to diameter and number analysis using the Multisizer 3. Each analysis in triplicate was performed based on $100 \mu \mathrm{L}$ of cells in electrolyte.

\section{Analysis of mitochondrial contents}

To investigate mitochondrial content in HCD1 and HCD3, a fluorescence microscopic analysis was performed using Mitotracker Green FM (Molecular Probes Inc., Eugene, OR, USA). Cells were stained with Mitotracker Green FM at a final concentration of $0.1 \mathrm{~g} \mathrm{~mL}^{-1}$ for $30 \mathrm{~min}$ at $25^{\circ} \mathrm{C}$. The stained cells were observed under a fluorescence microscope (BX53; Olympus Co., Tokyo, Japan) and images were taken using a CCD camera (DP73; Olympus Co.). Fluorescence intensity of cells was determined using ImageJ (http://imagej.nih.gov).

\section{Insertional mutant library construction}

To screen for insertional mutant strains with HCD phenotype, insertional mutants were constructed. Briefly, CC503 cultured in TAP medium were harvested and transformed with a $1.17 \mathrm{~kb}$ Ble gene fragment which was PCR-amplified from the plasmid pSP124S (Lumbreras et al. 1998) using the primers: Ble_1F (5'-TGGAAGCT TAAATGCCAGAAGGAGCGCAGCC-3') and Ble_1174R (5'-CCGAGCTCAGCTTCAAATACGCCCAGCCCG-3') (for nucleotide sequence of PCR fragment of the Ble expression cassette) (see Supplementary Fig. S1). Transformation was performed via electroporation as previously described (Shimogawara et al. 1998). The transformants were selected on TAP agar plates supplemented with 2.5 $\mu \mathrm{g} \mathrm{mL}^{-1}$ of zeocin (Invitrogen, Thermo Fisher Scientific Co., Waltham, MA, USA).

\section{Genetic screening for HCD insertional strains through continuous subcultures}

Over 1,000 transformed colonies were picked and pooled into a flask containing HS medium (Sueoka 1960) supplemented with $2.5 \mu \mathrm{g} \mathrm{mL}^{-1}$ zeocin and grown photoautotrophically to $\mathrm{OD}_{750}>1.0$ under continuous illumination of $50 \mu \mathrm{mol}$ photons $\mathrm{m}^{-2} \mathrm{~s}^{-1}$ and $2 \% \mathrm{CO}_{2}$ bubbling. The pool of mutants were subsequently used to seed three flasks to $\mathrm{OD}_{750}=\sim 0.15$. Continuous subcultures of stationary-phase cells were carried out for 30 cycles. Cell samples of the initial pool of mutant strains (0 subculture), the 10th, 20th, 30th cycles of subcultures were subjected to restriction fragment length polymorphism (RFLP) analysis.

\section{RFLP analysis}

To investigate growth of various insertional mutant strains during continuous subcultures, RFLP analysis of Ble sequences was performed. Briefly, cells were lysed using CTAB buffer $(2 \% \mathrm{w} / \mathrm{v}$ cetyltrimethyl ammonium bromide, $100 \mathrm{mM}$ Tris-HCl pH 6, $50 \mathrm{mM}$ ethylenediaminetetraacetic acid $\mathrm{pH}$ 7, $1.5 \mathrm{M} \mathrm{NaCl}, 50 \mathrm{mM}$ dithiothreitol) and genomic DNA molecules were subsequently extracted with phenol : chloroform. Probe for detection of Ble sequences was PCR amplified using primers Ble_E2_F (5'-TGGCCAAGCTGACCAGCGCCGTTCC-3') and Ble E3_R (5'-CCTCCGACCACTCGGCGTACAGC-3') on plasmid pSP124S (Lumbreras et al. 1998) as template (DIG Probe Synthesis Kit; Roche, Basel, Switzerland). Southern blotting analyses were performed according to manufacturer's instruction with buffers and solutions provided. The $\alpha$-DIG-AP was diluted $1: 15,000$ and washing buffer composition was modified to $0.1 \mathrm{M}$ maleic acid, $3 \mathrm{M}$ $\mathrm{NaCl}, 0.3 \%$ Tween 20, pH 8.0 to optimise the DIG detection step (Engler-Blum et al. 1993).

\section{Inverse-PCR analysis for identification of ge- nomic sequences flanking to insertion locus}

To identify the sequences flanking to the Ble insert, inverse-PCR methodology (Ochman et al. 1988) was utilized. Briefly, mutant genomic DNA was subjected to a single restriction enzyme digestion using one of restriction enzymes including but not limited to NheI, NdeI, and PstI. T4 DNA ligase (Life Technologies, Thermo Fisher Scientific Co.) was used to circularize the digested DNA. The resulting circularized DNA was used as template for PCR amplification of genomic DNA flanking 
to Ble insert using primers Ble_E2_R (5'-CCGGAACG GCGCTGGTCAGCTTG-3') and Ble_E3_F (5'-CTGCGT GCACTTCGTGGCCGAGG-3'). Flanking genomic DNA sequences derived from the inversely amplified DNA fragment were subjected to BLAST analysis against the genomic sequences of $C$. reinhardtii using the Phytozome tools (https://phytozome.jgi.doe.gov). Insertion locus in HCD1 and HCD3 was further validated using sequencespecific PCR analysis (see below).

\section{Sequence-specific PCR analysis}

To validate insertion locus in HCD mutants, sequencespecific PCR assay was performed using primer pairs of HCD1-F and HCD1-R (5'-CCGTACATGCCGCACAGGA AGCCTAGGTAGCG-3' and 5'-CCGAAGGCTGCACGTCC ACTGACCTGGATTG-3') for HCD1 and primer pairs of HCD3-F and HCD3-R (5'-CTCGACCATAAGGCGCCCGGC GTTTGAGTT-3' and 5'-GCCTTCAACGACGCGAGGTTCA ATACAGTGGAGC-3') for HCD3. PCR analysis was carried out using $2 \times$ Taq Master Mix (Probegene Lte., Xuzhou, China) under the conditions of $95^{\circ} \mathrm{C}$ for $60 \mathrm{~s} ; 95^{\circ} \mathrm{C}$ for $30 \mathrm{~s}, 64^{\circ} \mathrm{C}$ for $30 \mathrm{~s}$, and $72^{\circ} \mathrm{C}$ for $80 \mathrm{~s}$, those steps for 30 cycles; $72^{\circ} \mathrm{C}$ for $10 \mathrm{~min}$. Nucleotide sequences flanking to the HCD1 and HCD3 loci are available in Supplementary Figs S2 \& S3, respectively.

To examine the potential fusion transcript at the insertion locus, reverse transcription PCR assay was performed. Primers specific to Ble transcript (exon 2) Ble_E2_F2 (5'-GGTTCTCCCGGGACTTCGTGGAGGACGA CTTC-3') and flanking sequences at the exon 1 of Cre10. g426350 HCD1_R2 (5'-GTGGATCTCGAACTGCAAGGC GCACTAGGTGC-3') for HCD1 and flanking sequences at the $5^{\prime}$-untranslated region (5'-UTR) of Cre13.g585600 HCD3_R2 (5'-TGGAGCTCGTTGGTGCAGGTAATGCCCT TGTG-3') for HCD3. Nucleotide sequences of the fusion transcript with Ble in HCD1 and HCD3 are available in Supplementary Figs S4 \& S5, respectively.

To test transcriptional levels of genes flanking to the insertion site, reverse transcription quantitative PCR (RTqPCR) is performed in triplicate. Total RNA is extracted using TRIzol reagent (Takara Bio Inc., Tokyo, Japan). Total RNA of $500 \mathrm{ng}$ is used for first strand cDNA synthesis using 500 ng total RNA with PrimeScript RT reagent kit with gDNA Eraser (Takara Bio Inc.) according to manufacturer's protocol. The resulting cDNA is 10-fold diluted and subjected to quantitative PCR using SYBR Premix Ex Taq II kit (Takara Bio Inc.) on a Roche LightCycler 480 instrument (Roche) with the condition as follows: initial denaturing for $30 \mathrm{~s}$ at $95^{\circ} \mathrm{C}$, followed by 40 cycles of $5 \mathrm{~s}$ at $95^{\circ} \mathrm{C}$ and $30 \mathrm{~s}$ at $58^{\circ} \mathrm{C}$. Nucleotide sequences of gene-specific primers are as follows: for Cre10.g426350, HCD1Lfwd 5'-GCCAGCGAACCTGCGACAAG-3' and HCD1L-rev 5'-GCAGCCAGGAGGGGAACAGC-3'; for Cre10.g426400, HCD1R-fwd 5'-GCGGCATGTACGGGCTCTTC-3' and HCD1R-rev 5'-CCTCGGGCGTCAGCTTGTCC-3'; for Cre13. g585600, HCD3L-fwd 5'-AAGGTTGGCTTTTCGGGTCC-3' and HCD3L-rev 5'-TGCTGTTGTTCGAGTTGGTGC-3'; for Cre13.g585651, HCD3R-fwd 5'-CTTAGCAGCGGTG GAAGAGC-3' and HCD3R-rev 5'-GGCGGTGTCCGGGT CAATCC- $3^{\prime}$. The TUB2 is used as control whose primers are TUB2-fwd $5^{\prime}$-CCCCCGCCTGCACTTCTTC- 3 ' and TUB2-rev 5'-GTCGGCGGCGCACATCAT-3'.

\section{Transcriptome assembly analyses}

Hi-Seq 2000 PE90 technique was used for transcriptomic analysis of HCD mutant cells. For this reason, cell samples were harvested and total RNA was extracted using Trizol reagent (Life Technology) according to manufacturer's instructions. Resulting total RNA was subsequently treated with RNase-free DNaseI (Life Technology) and then column purified (RNeasy mini kit; Qiagen, Hilden, Germany). cDNA library construction for sequencing was performed in duplicate or triplicate by AIT Biotech (Singapore).

Approximately $\sim 22$ million good reads (90 nts in length) in each sample were subjected to Trimmomatic analysis (Bolger et al. 2014) in which 12 and 5 nucleotides at $5^{\prime}$ and $3^{\prime}$-ends (respectively) were trimmed. The resulting reads were subjected to TopHat analysis (Trapnell et al. 2009) to map reads based on reference genome of $C$. reinhardtii ver. 5.5 at Phytozome (https://phytozyme.jgi. goe.gov). Transcript abundances and differential transcription were obtained by using CuffLink and CuffDif analyses (Trapnell et al. 2009).

Meanwhile, the trimmed reads were also subjected to de novo assembly of transcripts using Trinity software aiming at finding novel transcripts at the insertion joint in HCD mutant strains when possible. Potential novel transcripts at the insertion locus were obtained by BLAST analysis (http://www.ncbi.nlm.nih.gov) of the Ble sequence and its flanking chromosomal sequences in HCD strains against the de novo assembled ESTs in respective mutant strains.

\section{GO functional enrichment analysis}

To investigate biological functions associated with the differentially transcribed genes in $\mathrm{HCD} 1$ and HCD3, gene 

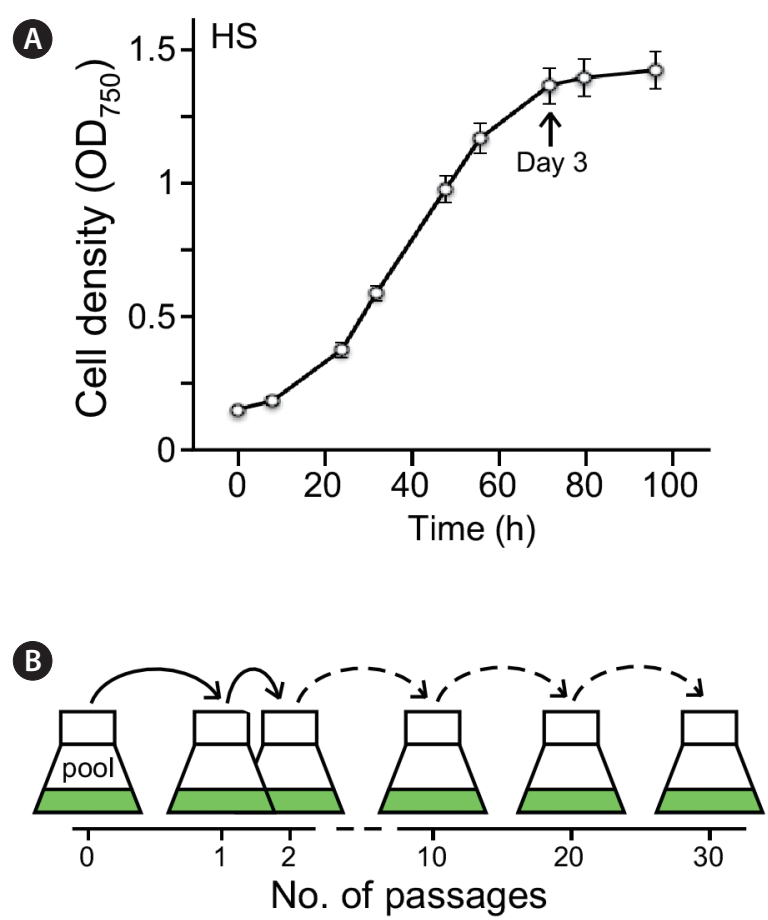

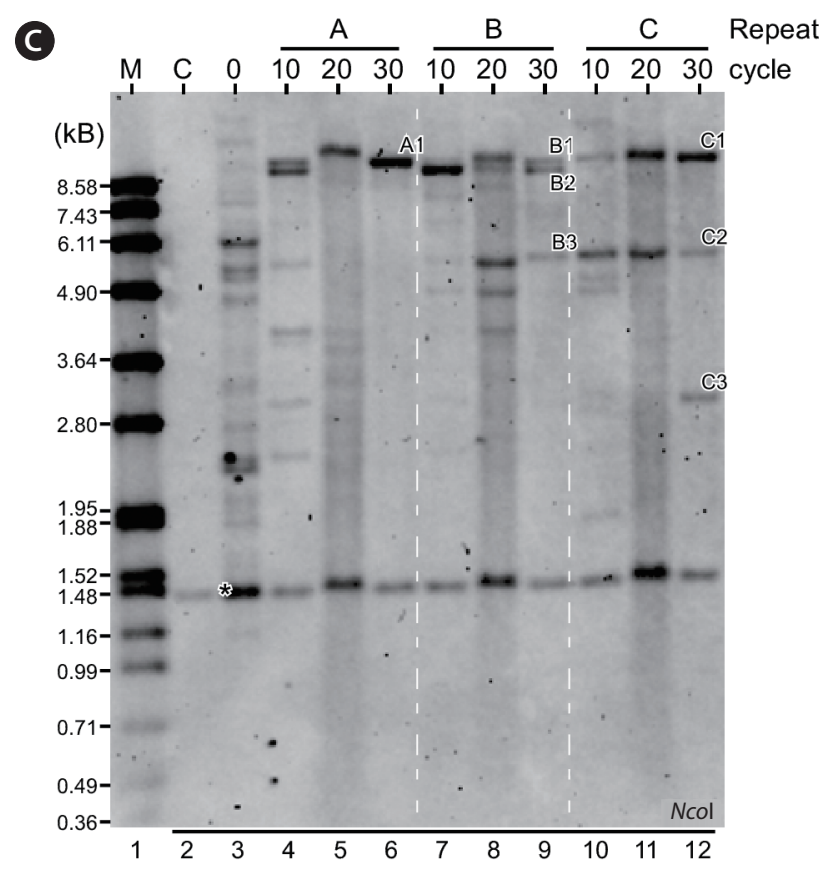

Fig. 1. Continuous subcultures enrich for insertional mutant strains exhibiting high cell-culture density phenotypes. (A) A typical growth curve of subcultures. After inoculation from day-3 early stationary-phase culture, subcultures were grown at the initial optical density at $750 \mathrm{~nm}\left(\mathrm{OD}_{750}\right)$ of $\sim 0.15$. (B) A schematic drawing of the continuous subculturing. (C) Enrichment of restriction fragment length polymorphism patterns of the continuous subculture experiments. Ncol digested genomic DNA was subjected to hybridization of DIG-labeled probes containing the Ble exon-2 and -3 sequences. Experiments $\mathrm{A}-\mathrm{C}$ are biological repeats. $\mathrm{M}$, marker; $\mathrm{C}, \mathrm{CC} 503$. Asterisk stands for endogenous background. Fragments $\mathrm{A} 1, \mathrm{~B} 1-3$, and 1 1-3 are enriched through continuous subcultures.

ontology (GO) enrichment analysis is performed in the subset of the top 500 differentially transcribed (upregulated or down regulated) genes using binomial test. GO biological functions is considered when a total number of associated genes is 14 or greater. The function is enriched when the fold of enrichment is 2 or greater and $\mathrm{p}$-value is less than 0.05 .

\section{Experimental replications and statistical analyses}

All experiments including growth curve analysis, growth rate, maximum cell dry weight, and chlorophyll contents of wild type and HCD insertional mutant strains were based on the analysis of 3 independent biological repeats. Significant differences of sample measurements from that of wild type were calculated using t-test.

Original RNA sequencing (RNA-seq) data for transcriptome assembly using reference mapping-based and de novo approaches are available in SRA database (http:// www.ncbi.nlm.nih.gov/sra) with an accession number SRP093432.

\section{RESULTS}

\section{Enrichment of HCD mutant strains in continuous subcultures}

To test whether quantitative genetic traits such as HCD would be enriched through continuous subcultures, we constructed over 1,000 individual insertional mutant strains generated through transformation of the PCRamplified Ble expression cassette using the template pSP124S plasmid (Lumbreras et al. 1998) into wild type strain CC503 cells. Equal amount of individual insertional mutant strains was pooled and cultivated in triplicate in $100 \mathrm{~mL}$ HS medium (Sueoka 1960) with 70-90 rpm shaking at room temperature under the continuous illumination of $50 \mu \mathrm{mol}$ photon $\mathrm{m}^{-2} \mathrm{~s}^{-1}$, bubbling with $\sim 2 \%$ $\mathrm{CO}_{2}$ (see Materials and Methods). Under this condition, the starting culture $\left(\sim 0.15 \mathrm{OD}_{750}\right)$ of the mixed mutant cells reached to $\sim 0.4, \sim 0.95, \sim 1.35$, and $\sim 1.45$ OD at day 1-4, respectively (Fig. 1A). Cells at day 3 entered the onset of stationary-phase and hence were used as inoculum to start the cycles of continuous subcultures (Fig. 1B). Sub- 

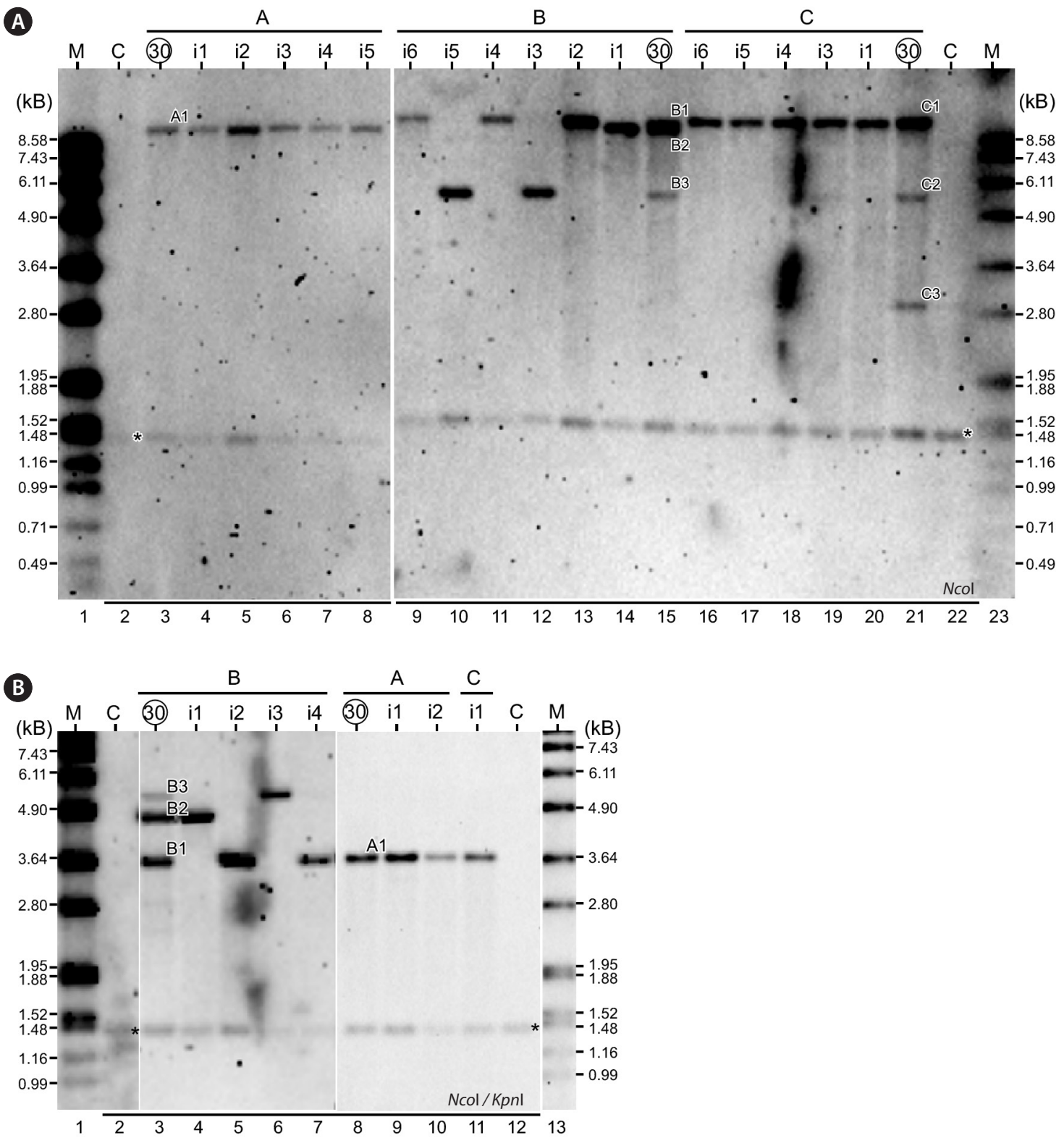

Fig. 2. Analysis of isolated high cell-culture density mutant strains. (A) Restriction fragment length polymorphism (RFLP) analysis of subcultures after 30 cycles and their isolates. Circled number on top indicates the subcultures after 30 cycles. Number prefixed with " $\mathrm{i}$ " indicates the individual isolates. Restriction enzyme used is shown at the bottom left. M, marker; C, CC503. (B) RFLP analysis with double digested genomic DNA.

cultures were continued for 30 cycles.

Mutant strains exhibiting HCD phenotypes would be expected to dominate the cultures containing over a thousand mutant populations after multiple rounds of subcultures. To investigate whether HCD mutant strains were gradually enriched in the continuous subcultures, RFLP pattern of the Ble expression cassette in NcoI digested genomic DNA derived from initial culture and subcultures after 10, 20, and 30 cycles were analyzed (Fig. 1C). A complex RFLP pattern was apparent in the initial culture containing the pool of a thousand insertional mutant strains (Fig. 1C, lane 3). We found that the complexity of RFLP patterns decreased upon increase of subculture cycles, suggesting that gradual enrichment of the HCD mutant strains occurred during continuous subcultures. In three independently repeated experiments A-C, 1-3 fragments (i.e., fragments A1, B1-3, and C1-3) in RFLP analysis were found after 30 cycles of subcultures. Strains bearing one of these fragments would be candidates of HCD strains. 

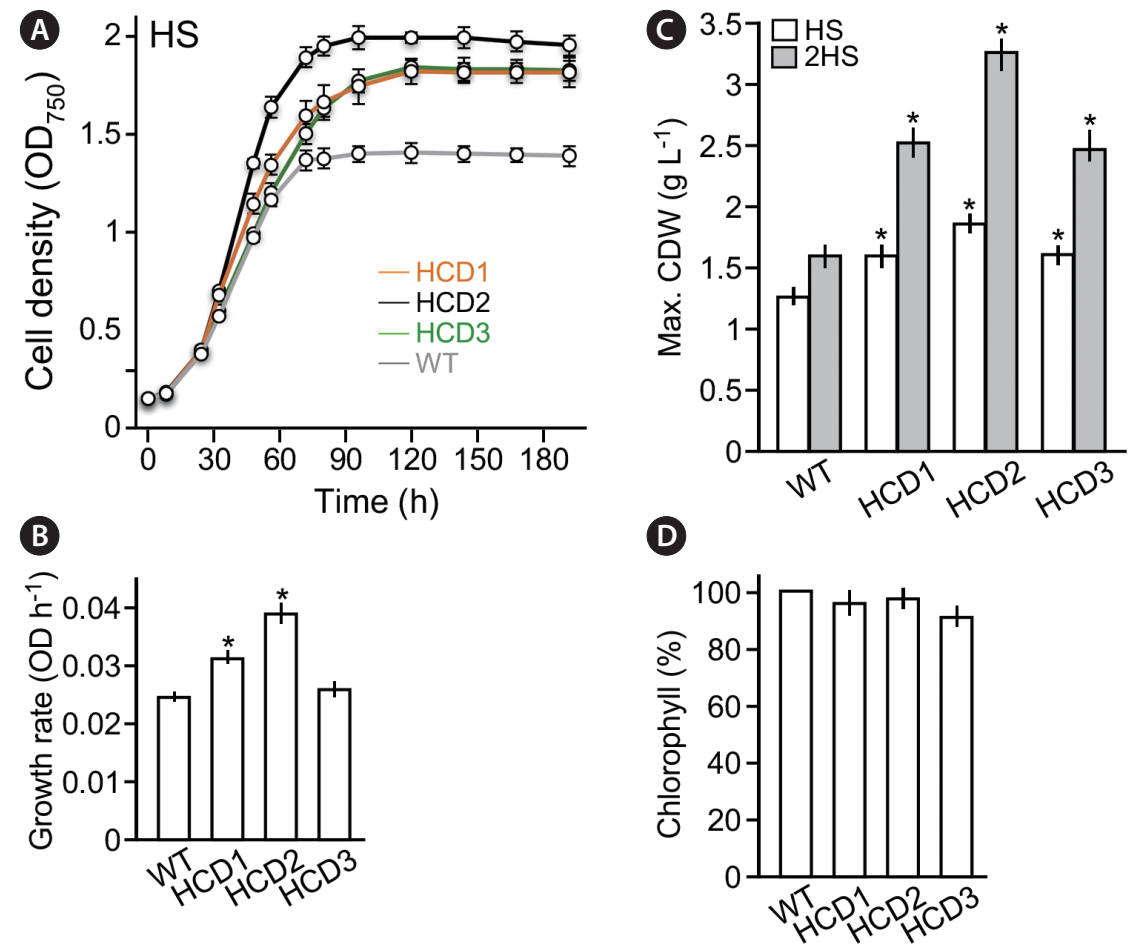

Fig. 3. Phenotypic analyses of high cell-culture density (HCD) mutant strains. (A) Growth curves. X-and Y-axis indicate time (h) and culture density (OD), respectively. Cells were cultivated in HS medium with a continuous illumination of 50 mol photon $\mathrm{m}^{-2} \mathrm{~s}^{-1}$. WT, wild type. (B) Logphase growth rate (between 24 and 56 h) of HCD mutant strains and WT. (C) Maximum cell dry weight (CDW) in various cultures. White and grey bars indicate cultures in $\mathrm{HS}$ and 2-time concentrated $\mathrm{HS}(2 \mathrm{HS})$ media with the illumination of $50 \mu \mathrm{mol}$ photon $\mathrm{m}^{-2} \mathrm{~s}^{-1}$. (D) Chlorophyll content in HCD strains compared to WT. Asterisk indicates significant difference from WT $(p<0.05)$.

\section{Isolation of enriched HCD mutant strains from cultures after 30 cycles of subcultures}

To isolate strains bearing one of the dominant RFLP fragments, cultures after 30 cycles of subcultures were plated out on solid HS plates for single colonies. Five to six randomly selected colonies from each repeated experiment were subjected to RFLP analysis using the $\mathrm{NcoI}$ digested genomic DNA. All 5 isolates from experiment A showed to have the fragment Al (Fig. 2A, lanes 4-8). In experiment $B$, isolate 2,4 , and 6 possessed the fragment B1 (Fig. 2A, lanes 9, 11, and 13), while isolate 3 and 5 contained the fragment B3 (Fig. 2A, lanes 10 and 12). One of the isolates in experiment $B$ showed to have the fragment B2 (Fig. 2A, lane 14). In experiment C, all 5 isolates showed to have the fragment $\mathrm{Cl}$ (Fig. 2A, lanes 16-20). None of the isolates tested was found to possess the fragment $\mathrm{C} 2$ or $\mathrm{C} 3$.

To confirm the isolates contained the enriched fragments after 30 cycles of continuous subcultures based on the RFLP analysis using $\mathrm{NcoI}$ single digested genomic
DNA, we performed the RFLP analysis using NcoI and $K p n I$ double digested genomic DNA. Although the sizes of enriched fragments altered in double digestion, relationship between isolates and its RFLP patterns remained identical to that of single digestion (Fig. 2B), supporting the RFLP analysis based on single $\mathrm{NcoI}$ digestion (see Fig. 2A). Hence, we designated the mutant strains containing the fragments B1 (isolates A1_i1-i5; B1_i2, i4, and i6; and C1_il and i3-i6), B2 (isolate B2_i1), and B3 (B3_i3 and i5) as HCD1, HCD2, and HCD3, respectively.

\section{HCD mutant strains exhibit high maximum cell- culture density phenotypes}

To investigate the maximum cell density of the HCD mutant strains, growth curves analyses were performed under photoautotrophic growth conditions (HS medium) with continuous illumination at $50 \mu \mathrm{mol}$ photon $\mathrm{m}^{-2} \mathrm{~s}^{-1}$ (Fig. 3A). We found that the growth rates (i.e., average growth rate at the log-phase from 24 to $56 \mathrm{~h}$ based on triplicate) of HCD1 and HCD2 but not HCD3 were 
significantly higher than that of wild type $(\mathrm{p}<0.05)$ (Fig. 3B). On the other hand, the maximum cell densities of HCD1, HCD2, and HCD3 in HS medium were $22-40 \%$ higher than that of wild type ( $\mathrm{p}<0.05$ ) (Fig. 3C, open bar). When cells were cultivated in 2-times concentrated HS medium (2HS medium), the maximum cell density of the HCD mutant strains were $50-100 \%$ higher than that of wild type $(\mathrm{p}<0.05)$ (Fig. 3C, grey bar).

To investigate the level of chlorophyll contents in HCD mutant strains, we determined the ratio of chlorophyll and cell dry weight. Percent of chlorophyll contents in cell dry weight of HCD mutant strains were not significantly lower than that of wild type (Fig. 3D), suggesting that the HCD phenotypes are unrelated to that of truncated light-harvesting antenna TLA mutants (Polle et al. 2003). This result is consistent with the notion that biomass yield of TLA mutant strains increases under growth conditions with high illumination such as $>500 \mu \mathrm{mol}$ photon $\mathrm{m}^{-2} \mathrm{~s}^{-1}$ (Polle et al. 2003) but not low illumination (e.g., $\sim 50 \mu \mathrm{mol}$ photon $\mathrm{m}^{-2} \mathrm{~s}^{-1}$ ).

\section{Identification of insertion loci in HCD mutant strains using inverse-PCR methodology}

Given that HCD1, HCD2, and HCD3 exhibited a single fragment in RFLP analysis of both single and double digestions (see Fig. 2), we assumed that only a single copy of the Ble expression cassette was inserted into the genome of individual HCD mutant strains. Hence, we mapped the insertion locus in HCD mutant strains using inverse-PCR analysis (Yoshioka et al. 2004). For this reason, genomic DNA of HCD mutant cells was subjected to single digestions of various restriction enzymes including but not limited to NheI, NdeI, and PstI that had no recognition site in the Ble cassette insert (Fig. 4A). The resulting DNA was then self-ligated with T4 DNA ligase and followed by PCR amplification using sequence-specific primers E2R (Ble exon 2 reverse) and E3F (Ble exon 3 forward) (see Materials and Methods). PCR fragments were successfully amplified in HCD1 and HCD3 but not HCD2 after self-ligation.

Sequencing analysis of the inverse-PCR fragments generated from HCD1 suggested that the Ble cassette was inserted in the first exon of Cre10.g426400 (Fig. 4B). We applied PCR analysis using sequence-specific primers flanking to the Ble insert to confirm its insertion locus in HCD1. We found that the identical pair of primers gave rise to a fragment of $\sim 1.1 \mathrm{~kb}$ in size using HCD1 genomic DNA as template, which was $\sim 850$ bp longer than the fragment of $\sim 250 \mathrm{bp}$ in size using wild type CC503 ge- nomic DNA as template (Fig. 4C). This result confirmed that the Ble cassette was inserted into the first exon of Cre10.g426400, splitting it into two parts: Cre10.g426400lel (or transcript containing the first part of exon 1) and Cre10.g426400-2el (or transcript containing the second part of exon 1). Sequencing analysis of the PCR fragment encompassing the HCD1 locus indicated that the Ble cassette was inserted in a reverse orientation into the first exon of Cre10.g426400 at positions of 141 and $145 \mathrm{nt}$ with a 3-nt deletion in Cre10.g426400 (Fig. 4D). The insert contained Ble cassette sequences from positions 81 to $900 \mathrm{nt}$ missing the entire $3^{\prime}$-UTR. Meanwhile, we found that a single nucleotide and trinucleotide were inserted in the left and right joints flanking the Ble insert, respectively.

Similarly, inverse-PCR analysis of HCD3 indicated that the Ble cassette was inserted into the intergenic region between Cre13.g585600 and Cre13.g585651 (Fig. 4E). PCR analysis using sequence-specific primers showed that fragments of $\sim 1.4 \mathrm{~kb}$ and $300 \mathrm{bp}$ in size were amplified in HCD3 and wild type (respectively), indicating a fragment of $\sim 1.1 \mathrm{~kb}$ in size was present at the insertion locus of HCD3 (Fig. 4F). Sequencing analysis of the PCR fragment encompassing the HCD3 locus indicated that most of the Ble cassette sequences were inserted into the intergenic region at positions from -52 and -63 nt with a deletion of $10 \mathrm{nt}$ based on the position of Cre13.g585600 (Fig. 4G). The Ble cassette sequences from positions 6 to $1,132 \mathrm{nt}$ (the whole Ble cassette was $1.152 \mathrm{~kb}$ in length out of the full-length PCR fragment of $1.174 \mathrm{~kb}$ ) were found in the insertion locus of HCD3. The left joint showed no nucleotide addition. On the other hand, a hexanucleotide were found in the right joint. These results indicated that part of the terminal sequences in the Ble cassette could be lose during insertion into genome. In addition, single and short stretches of nucleotides such as trinucleotide and hexanucleotide could be added during random insertion.

\section{Transcription enhancement of the disrupted partial transcript and the Ble-fusion transcript at the HCD1 locus}

To investigate whether the transcription of genes flanking to the insert at the HCD1 locus was disrupted in the mutant strain, we performed transcriptional profiling analysis of the HCD1 and wild type CC503 strain (see Materials and Methods). In this analysis, RNA samples was subjected to library construction for sequencing using illumine HiSeq technology in triplicate (see Materials and Methods). Transcriptomes was assembled us- 

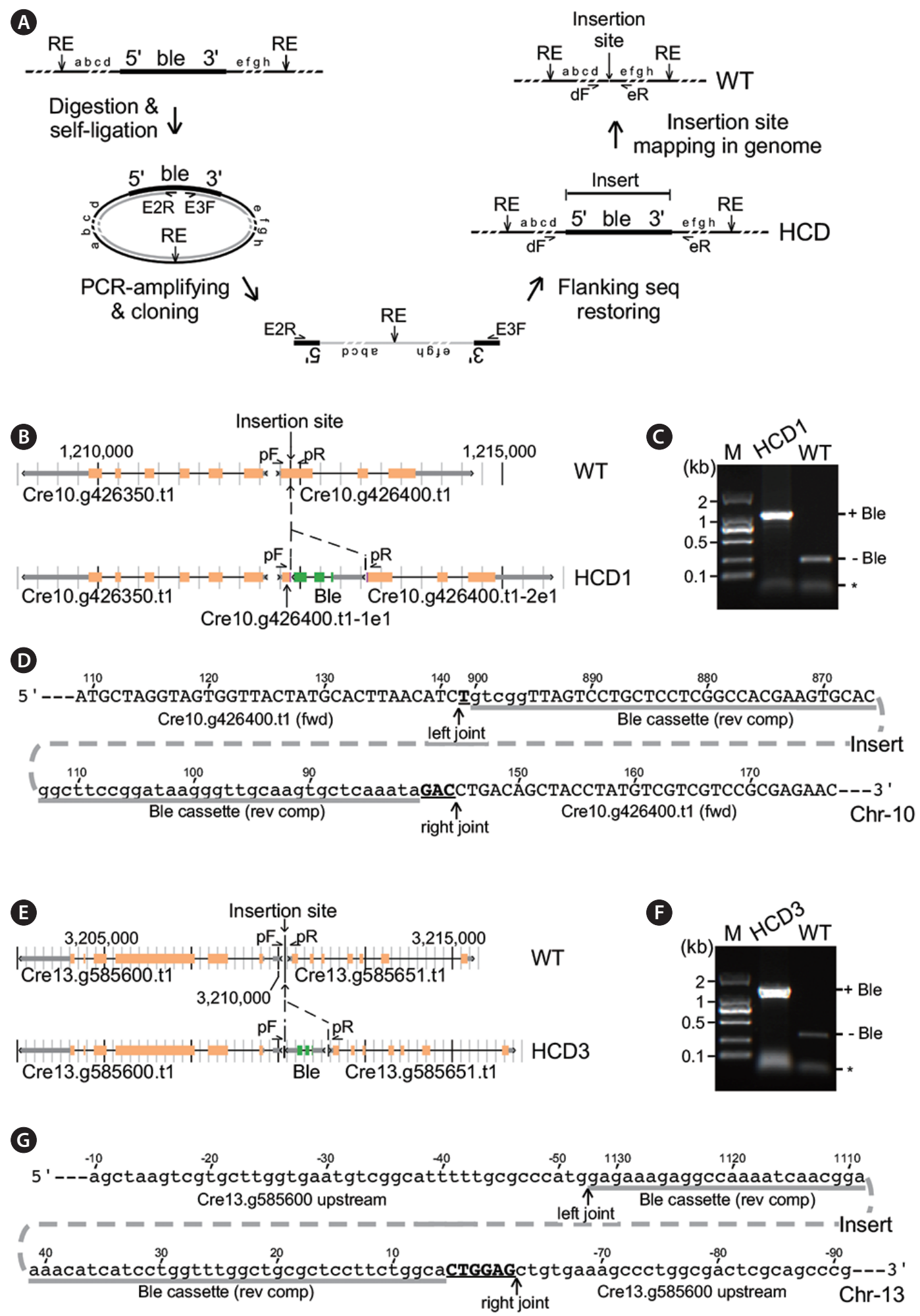

Fig. 4. Identification of insertion sites in high cell-culture density (HCD) 1 and HCD3. (A) Schematic drawing of the inverse polymerase chain reaction (inverse-PCR) analysis. WT, wild type. (B) Physical map of the insertion locus in HCD1 cells. (C) PCR assay for Ble-sequences (+Ble) at the insertion site in HCD1. (D) Nucleotide sequences at the insertion site in HCD1. Upper and lower cases indicate coding and noncoding sequences based on reference genome. Sequence coordinate is based on the PCR fragment of the Ble expression cassette and transcription unit of Cre10. g426400.t1. (E) Physical map of the insertion locus in HCD3 cells. (F) PCR assay for Ble-sequences at the insertion site in HCD3. (G) Nucleotide sequences at the insertion site in HCD3. The display is identical to (D). 

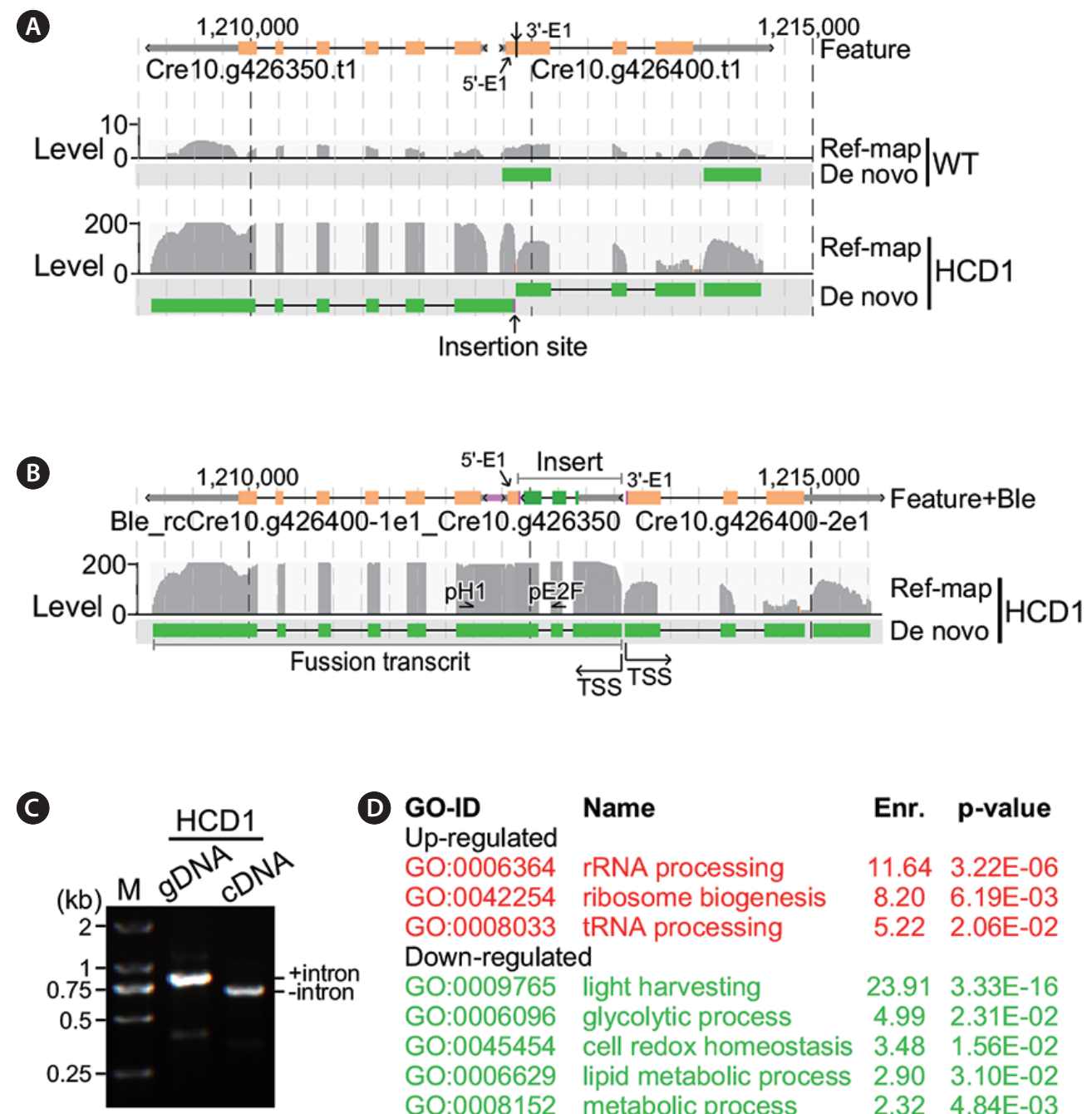

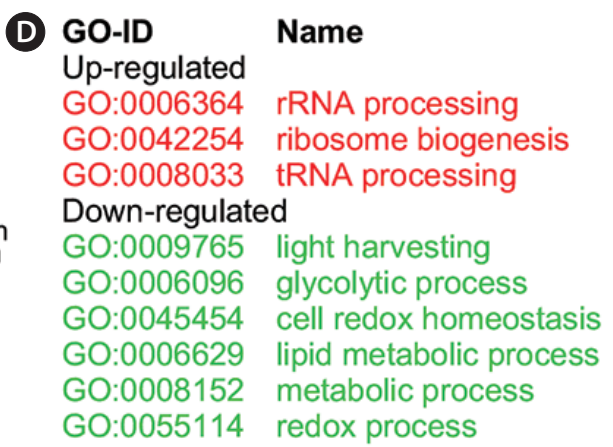

$\begin{array}{cc}\text { Enr. } & \text { p-value } \\ 11.64 & 3.22 \mathrm{E}-06 \\ 8.20 & 6.19 \mathrm{E}-03 \\ 5.22 & 2.06 \mathrm{E}-02 \\ & \\ 23.91 & 3.33 \mathrm{E}-16 \\ 4.99 & 2.31 \mathrm{E}-02 \\ 3.48 & 1.56 \mathrm{E}-02 \\ 2.90 & 3.10 \mathrm{E}-02 \\ 2.32 & 4.84 \mathrm{E}-03 \\ 2.13 & 3.16 \mathrm{E}-04\end{array}$

Fig. 5. Analysis of flanking gene transcription at the insertion site in high cell-culture density 1 (HCD1). (A) Transcription of genes at the insertion site in HCD1 based on sequences without the insert. Genome features and coordinates are indicated on top. Transcripts detected in reference mapping-based (Ref-map) and de novo (De novo) assembly analyses are indicated on the left. Level of transcripts (in fragments per kilobase of exon per million fragments mapped) is indicated on the right. An arrow indicates the insertion site. WT, wild type. (B) Transcription of genes at the insertion site in HCD1 based on sequences containing the insert. The display is identical to (A). TSS, transcription start site. (C) Reverse transcription polymerase chain reaction assay on HCD1 genomic DNA (gDNA) and cDNA. M, marker. (D) Functional enrichment analysis. Upregulated and downregulated gene ontology (GO) functions are shown in red and green, respectively.

ing reference-based methodology (Trapnell et al. 2009) (Supplementary Table S1). In wild type cells, transcription levels of Cre10.g426400 at the HCD1 locus was 6.26 fragments per kilobase of exon per million fragments mapped (FPKM), based on the average of three repeated measurements (all measurements of transcriptional levels were derived from the average of three repeats) (Fig. $5 \mathrm{~A}$, upper track). We found that the transcription level of Cre10.g426400 was 39.66 FPKM in HCD1 cells, which was 6.33 -fold higher than that of wild type cells $(\mathrm{p}=2.4 \mathrm{e}$ 04) (Fig. 5A, lower track). Meanwhile, we found that the transcription level of Cre10.g426350, upstream of Cre10. g426400, was 3.89 FPKM in wild type. The level of Cre10. g426350 was increased to 170.89 FPKM in HCD1, indicating that Ble cassette insertion induced the transcription of Cre10.g426350 by 44-fold.

We hypothesized the promoter P-RBCS2 in the Ble cassette was responsible for the bi-directional activation of transcription at the HCD1 locus. To test this possibility, we performed the de novo assembly (Grabherr et al. 2011) of transcriptomes of HCD1 mutant. Analysis of the de novo assembled transcripts containing the HCD1 flank- 

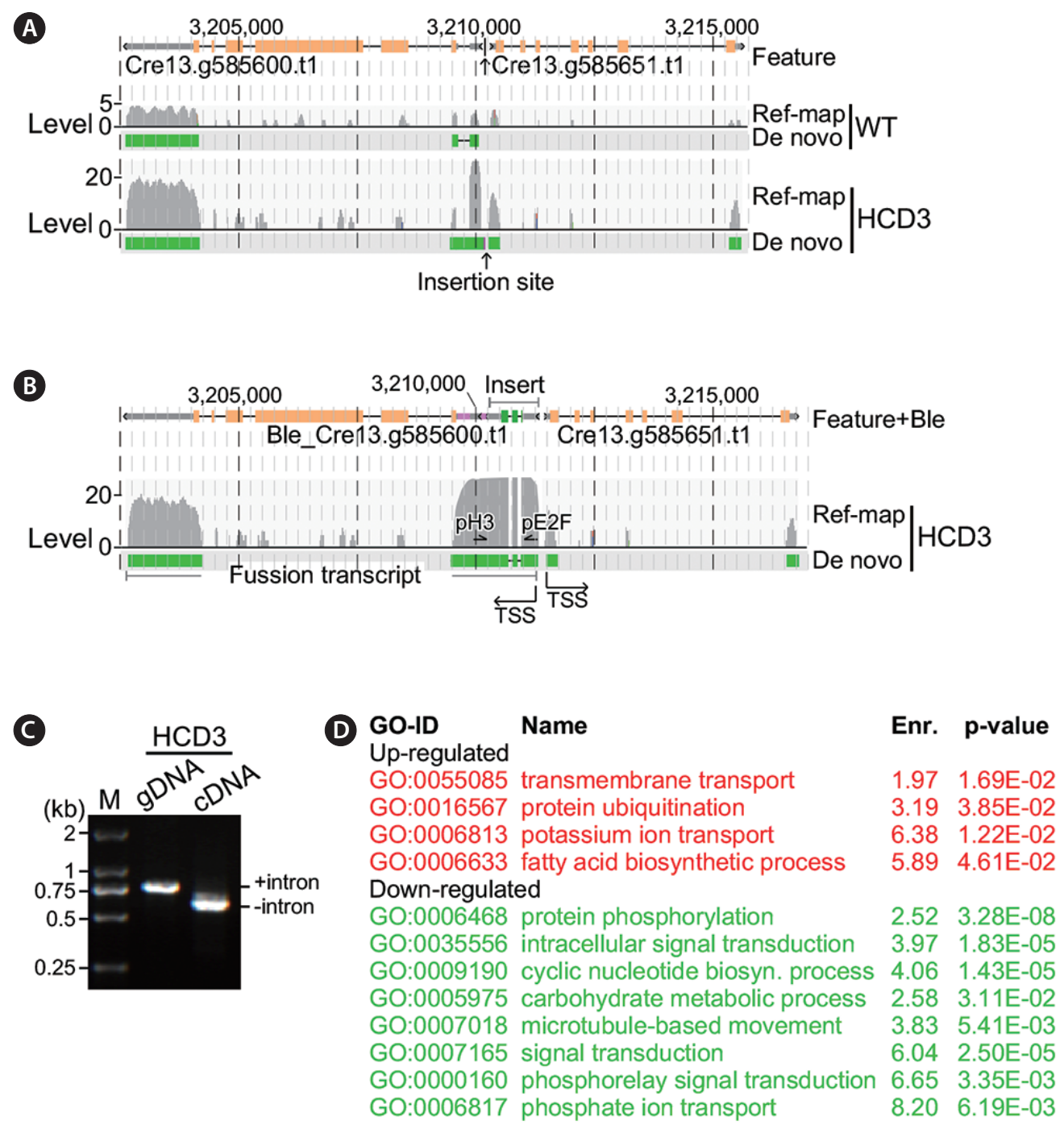

Fig. 6. Analysis of flanking gene transcription at the insertion site in high cell-culture density 3 (HCD3). The display is identical to Fig. 5. (A) Transcription of genes at the insertion site in HCD3 based on sequences without the insert. WT, wild type. (B) Transcription of genes at the insertion site in HCD3 containing the insert. TSS, transcription start site. (C) Reverse transcription polymerase chain reaction assay on HCD3 genomic DNA (gDNA) and cDNA. M, marker. (D) Functional enrichment analysis. Upregulated and downregulated gene ontology (GO) functions are shown in red and green, respectively.

ing gene sequences indicated that the transcription start site (TSS) of Cre10.g426400-2el (i.e., transcript containing the second part of exon 1) was located at boundary of the right joint. On the other hand, reverse-complementary sequences of Cre10.g426400-1el was found in a fusion transcript containing Ble, reverse-complementary (rc) Cre10.g426400-1e1, and Cre10.g426350 sequences (Fig. $5 \mathrm{~B})$. To investigate the presence of the fusion transcript containing Ble, rcCre10.g426400-1e1, and Cre10.g426350 sequences, we performed PCR analysis using sequencespecific primers located at Ble exon 2 and Cre10.g426350 exon 1 using either HCD1 genomic DNA or cDNA as tem- plate. In this case, the two primers would amplify a fragment encompassing the intron 2 of $145 \mathrm{bp}$ in size located between Ble exon 2 and Cre10.g426350 exon 1 in the HCD1 genomic DNA but not cDNA. The result showed while a fragment of $\sim 850 \mathrm{bp}$ in size was amplified using genomic DNA as template, a fragment of $\sim 700 \mathrm{bp}$ in size was amplified using cDNA template, supporting the existence of the fusion transcript containing Ble, rcCre10. g426400-1e1, and Cre10.g426350 sequences (Fig. 5C).

Subsequently, we performed functional enrichment analysis in top 500 differentially transcribed genes in HCD1 (see Materials and Methods). Analysis 

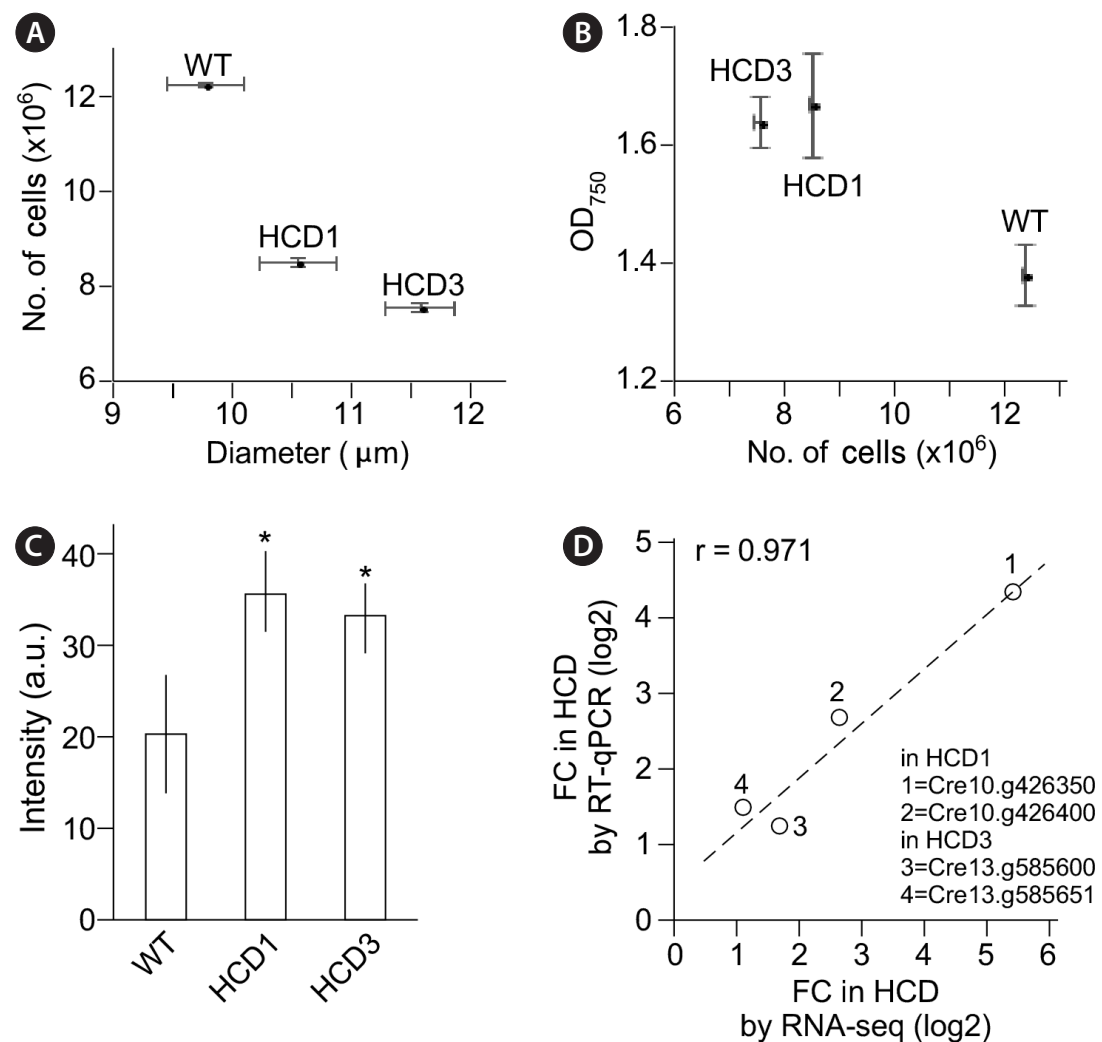

Fig. 7. Subtle phenotypes associated with high cell-culture density (HCD) 1 and HCD3 mutants. (A) HCD mutant cells exhibit large size of cells than does the wild type (WT). (B) HCD mutant cultures exhibit higher optical density (OD) value but lower cell counts per milliliter than does the WT. (C) HCD mutant cells exhibit higher mitotracker fluorescence signals than does the WT. Asterisk indicates significant difference from WT $(p<0.05)$. (D) Genes at the insertion site in HCD mutant cells whose transcription level changes determined by RNA sequencing (RNA-seq) is correlated with reverse transcription quantitative polymerase chain reaction (RT-qPCR). FC, fold-change.

indicated that functions related with ribosomal biogenesis (GO:0006364, GO:0042254, and GO:0008033) were enriched in the top 500 significantly upregulated genes in HCD1 (Fig. 5D). There were six GO functions (GO:0009765, GO:0006096, GO:0045454, GO:0006629, GO:0008152, and GO:0055114) were found to be enriched in the top 500 significantly downregulated genes in HCD1, two of which were related to the putative thioredoxin function of the disrupted Cre10.g426400.

\section{Intergenic insertion induces transcription of flanking genes in HCD3 locus}

To investigate alteration of transcriptions at the HCD3 insertion site, we performed reference-based and de novo transcriptome analyses of HCD3 mutant strain and found that the transcriptional levels of Cre13.g585600 and Cre13.g585651 were increased by 3.31-fold (8.57 FPKM vs. 3.31 FPKM, $\mathrm{p}=2.4 \mathrm{e}-04$ ) and 2.21-fold (2.66 FPKM vs. 1.21 FPKM, $\mathrm{p}=4.9 \mathrm{e}-02$ ) in HCD3 cells compared to that of wild type (Fig. 6A). Analysis of de novo transcripts containing the flanking gene sequences indicated that the TSS of Cre13.g585651 was not altered in HCD3 compared to that of wile type (Fig. 6B). On the other hand, Cre13.g585600 transcript was found to be fused with Ble sequences, that was inserted at the upstream of Cre13.g585600 with the same orientation. To confirm the presence of the fusion transcript between Ble and Cre13.g585600, we performed PCR analysis using sequence-specific primers located at Ble exon 2 and Cre13.g585600 exon 1 using either HCD3 genomic DNA or cDNA as template. It was clear that the intron sequence was excised in cDNA or transcript but not in genomic DNA (Fig. 6C).

Functional enrichment analysis indicated that, in HCD3, four GO functions (GO:0055085, GO:0016567, GO:0006813, GO:0006633) were enriched in the top 500 significantly upregulated genes (Fig. 6D). Lon protease was proposed to be linked to the proteasome function (Erjavec et al. 2013). While Cre13.g426400 encoded a putative Lon protease, upregulation of protein ubiquitina- 
tion function is consistent with its upregulation in HCD3. On the other hand, eight GO functions (GO:0006468, GO:0035556, GO:0009190, GO:0005975, GO:0007018, GO:0007165, GO:0000160, and GO:0006817) were enriched in the top 500 significantly downregulated genes in HCD3.

\section{Phenotypes associated with HCD1 and HCD3 mutants}

We found that, at the onset of stationary phase (e.g., 96 $\mathrm{h}$ after growth), the median diameter of $\mathrm{HCD} 1$ and HCD3 cells was 10.6 and $11.6 \mu \mathrm{m}$, which was 7 and $17 \%$ bigger than that of wild type $(9.9 \mu \mathrm{m})(\mathrm{p}=0.0012$ and $\mathrm{p}=8.24 \mathrm{e}$ 05 , respectively) (Fig. 7A \& B). To test whether the size of mitochondria in HCD1 mutant cells altered as well, cells were subjected to mitotracker fluorescence dye staining (see Materials and Methods). The mitochondrial sizes were approximated by the average fluorescence signals. To this end, we found that the average fluorescence signals in HCD1 and HCD3 were 53.7 and 33.1 (arbitrary unit), which was 1.77 -fold and 1.64-fold higher than 20.2 a.u. of wild type $(p=2 e-06$ and $p=1 e-05$, respectively $)$ (Fig. 7C).

Transcription levels of genes at the insertion site in HCD1 or HCD3 were found to be altered in RNA-seq analysis (see Figs 5A \& 6A). To validate the change, RTqPCR was performed. We found that the fold changes of transcription levels of at the insertion site in HCD1 or HCD3 in RT-qPCR and RNA-seq analyses were well correlated with a correlation coefficient of 0.971 (Fig. 7D).

\section{DISCUSSION}

Increasing microalgal maximum cell-culture density is one of the ways for improving its biomass productivity. Early works have shown that $C$. reinhardtii cells with reduced size of light-harvesting antenna or lowered chlorophyll content exhibit increased cell-culture density under high-illumination growth conditions (Polle et al. 2003). In this study, we show that $C$. reinhardtii mutant strains without lowering the chlorophyll content display the HCD phenotype under low-illumination condition (see Fig. 3).

It has been proposed that only a small fraction of the light harvested by algal cells with large-sized antenna is converted to chemical energy under high-illumination (Polle et al. 2003). Hence, small-sized antenna allows more cells to access light and thus increases the efficiency of light usage. In this study, we show that in HCD1, the insert disrupted the intragenic sequences of Cre10.g426400, leading to the increase of transcription levels of the truncated Cre10.g426400-2e1 transcript and the fusion transcript containing Ble, rcCre10.g4264001e1, and Cre10.g426350 (see Fig. 5). While the function of Cre10.g426350 is unknown, Cre10.g426400 encodes a putative thioredoxin. Transcription profile analysis indicates that ribosomal biogenesis functions (GO:0006364, GO:0042254, and GO:0008033) are enriched in the top significantly upregulated genes in HCD1, consistent with its high biomass yield. Additionally, redox processes (GO:0045454 and GO:0055114) are enriched in the most downregulated genes, in agreement with the disruption of the putative thioredoxin gene Cre10.g426400.

In HCD3, the Ble cassette was inserted at the intergenic region between Cre13.g585600 and Cre13. g585651, leading to the transcription level increase of Cre13.g585651 and a fusion transcript containing Ble and Cre13.g585600 (see Fig. 6). Though the function of Cre13.g585600 is unknown, Cre13.g585651 encoded a putative mitochondrial ATP-dependent Lon protease. Products of both Cre10.g426400 in HCD1 and Crel3. g585651 in HCD3 are potentially linked to mitochondrial function (Kang et al. 2002, Folda et al. 2016). We show that both HCD1 and HCD3 exhibit elevated mitotracker fluorescence staining, suggesting a link to mitochondrial function in increased cell density phenotype. Hence, we propose that abnormality of mitochondrial function in HCD1 and HCD3 play a role in increased cell-culture density under low-illumination condition.

In this study, we show that mutant strains exhibiting quantitative traits of HCD are enriched from the pool of the randomly mutated strains through continuous subcultures. Analysis of clonal HCD isolates confirms their high biomass yield under low-illumination growth conditions. Molecular analysis indicates that transcription of flanking genes is upregulated by the intragenic and intergenic insertions in HCD1 and HCD3, respectively. In both cases, transcriptionally enhanced genes flanking to the insertion site with or without intact sequences are potentially linked to the mitochondrial functions (Kang et al. 2002, Folda et al. 2016). We propose that by using continuous subcultures methodology, more HCD strains can be enriched from a large pool of insertional mutant strains. Analysis of novel HCD strains will allow gaining insight into mechanisms for regulation of cell-culture density under various illumination conditions in C. reinhardtii and other green algal species. 


\section{ACKNOWLEDGEMENTS}

The authors would like to thank L. Fang and S. Qi for their technical assistance and to an anonymous reviewer's comment that has greatly improved the quality of this manuscript. This work was partly supported by grants from National Natural Science Foundation of China, China (Grant no.: 31571392) and Zhoushan Municipal Science and Technology Bureau, China (Grant no.: 2015C51024; 2015C51025) to J. Liu and a grant from Singapore A-STAR Joint Council Office, Singapore (Grant no.: 11/03/FG/07/06) to J. Liu and Y. Chew. L. Thung was a recipient of the post-doctoral fellowship from Singapore A-STAR Joint Council Office, Singapore.

\section{SUPPLEMENTARY MATERIAL}

Supplementary Table S1. Transcription profiles of high cell-culture density (HCD) 1, HCD3, and the wild type (http://e-algae.org).

Supplementary Fig. S1. Nucleotide sequence of polymerase chain reaction amplified Ble expression cassette (http://e-algae.org).

Supplementary Fig. S2. Nucleotide sequences encompassing the high cell-culture density 1 (HCD1) locus (http://e-algae.org).

Supplementary Fig. S3. Nucleotide sequences encompassing the high cell-culture density 3 (HCD3) locus (http://e-algae.org).

Supplementary Fig. S4. Partial nucleotide sequences encompassing the excised Ble intron 2 site in the fusion transcript in high cell-culture density 1 (HCD1) (http://ealgae.org).

Supplementary Fig. S5. Partial nucleotide sequences encompassing the excised Ble intron 2 site in the fusion transcript in high cell-culture density 3 (HCD3) (http://ealgae.org).

\section{REFERENCES}

Bolger, A. M., Lohse, M. \& Usadel, B. 2014. Trimmomatic: a flexible trimmer for Illumina sequence data. Bioinformatics 30:2114-2120.

Cheng, X., Liu, G., Ke, W., Zhao, L., Lv, B., Ma, X., Xu, N., Xia, X., Deng, X., Zheng, C. \& Huang, K. 2017. Building a multipurpose insertional mutant library for forward and reverse genetics in Chlamydomonas. Plant Methods 13:36.

Dent, R. M., Haglund, C. M., Chin, B. L., Kobayashi, M. C.
\& Niyogi, K. K. 2005. Functional genomics of eukaryotic photosynthesis using insertional mutagenesis of Chlamydomonas reinhardtii. Plant Physiol. 137:545556.

Engler-Blum, G., Meier, M., Frank, J. \& Müller, G. A. 1993. Reduction of background problems in nonradioactive northern and southern blot analyses enables higher sensitivity than 32P-based hybridizations. Anal. Biochem. 210:235-244.

Erjavec, N., Bayot, A., Gareil, M., Camougrand, N., Nystrom, T., Friguet, B. \& Bulteau, A. L. 2013. Deletion of the mitochondrial Piml/Lon protease in yeast results in accelerated aging and impairment of the proteasome. Free Radic. Biol. Med. 56:9-16.

Folda, A., Citta, A., Scalcon, V., Calì, T., Zonta, F., Scutari, G., Bindoli, A. \& Rigobello, M. P. 2016. Mitochondrial thioredoxin system as a modulator of cyclophilin D redox state. Sci. Rep. 6:23071.

González-Ballester, D., de Montaigu, A., Higuera, J. J., Galván, A. \& Fernandez, E. 2005. Functional genomics of the regulation of the nitrate assimilation pathway in Chlamydomonas. Plant Physiol. 137:522-533.

Grabherr, M. G., Haas, B. J., Yassour, M., Levin, J. Z., Thompson, D. A., Amit, I., Adiconis, X., Fan, L., Raychowdhury, R., Zeng, Q., Chen, Z., Mauceli, E., Hacohen, N., Gnirke, A., Rhind, N., di Palma, F., Birren, B. W., Nusbaum, C., Lindblad-Toh, K., Friedman, N. \& Regev, A. 2011. Fulllength transcriptome assembly from RNA-Seq data without a reference genome. Nat. Biotechnol. 29:644652.

Harris, E. H. 1989. The Chlamydomonas sourcebook: a comprehensive guide to biology and laboratory use. Academic Press, San Diego, CA, 780 pp.

Harris, E. H. 2001. Chlamydomonas as a model organism. Annu. Rev. Plant Physiol. Plant Mol. Biol. 52:363-406.

Kang, S. G., Ortega, J., Singh, S. K., Wang, N., Huang, N. N., Steven, A. C. \& Maurizi, M. R. 2002. Functional proteolytic complexes of the human mitochondrial ATPdependent protease, hClpXP. J. Biol. Chem. 277:2109521102.

León-Bañares, R., González-Ballester, D., Galván, A. \& Fernández, E. 2004. Transgenic microalgae as green cellfactories. Trends Biotechnol. 22:45-52.

Lumbreras, V., Stevens, D. R. \& Purton, S. 1998. Efficient foreign gene expression in Chlamydomonas reinhardtii mediated by an endogenous intron. Plant J. 14:441-447.

Merchant, S. S., Prochnik, S. E., Vallon, O., Harris, E. H., Karpowicz, S. J., Witman, G. B., Terry, A., Salamov, A., FritzLaylin, L. K., Maréchal-Drouard, L., Marshall, W. F., Qu, L. -H., Nelson, D. R., Sanderfoot, A. A., Spalding, M. H., 
Kapitonov, V. V., Ren, Q., Ferris, P., Lindquist, E., Shapiro, H., Lucas, S. M., Grimwood, J., Schmutz, J., Cardol, P., Cerutti, H., Chanfreau, G., Chen, C. -L., Cognat, V., Croft, M. T., Dent, R., Dutcher, S., Fernández, E., Ferris, P., Fukuzawa, H., González-Ballester, D., González-Halphen, D., Hallmann, A., Hanikenne, M., Hippler, M., Inwood, W., Jabbari, K., Kalanon, M., Kuras, R., Lefebvre, P. A., Lemaire, S. D., Lobanov, A. V., Lohr, M., Manuell, A., Meier, I., Mets, L., Mittag, M., Mittelmeier, T., Moroney, J. V., Moseley, J., Napoli, C., Nedelcu, A. M., Niyogi, K., Novoselov, S. V., Paulsen, I. T., Pazour, G., Purton, S., Ral, J. -P., Riaño-Pachón, D. M., Riekhof, W., Rymarquis, L., Schroda, M., Stern, D., Umen, J., Willows, R., Wilson, N., Zimmer, S. L., Allmer, J., Balk, J., Bisova, K., Chen, C. -J., Elias, M., Gendler, K., Hauser, C., Lamb, M. R., Ledford, H., Long, J. C., Minagawa, J., Page, M. D., Pan, J., Pootakham, W., Roje, S., Rose, A., Stahlberg, E., Terauchi, A. M., Yang, P., Ball, S., Bowler, C., Dieckmann, C. L., Gladyshev, V. N., Green, P., Jorgensen, R., Mayfield, S., Mueller-Roeber, B., Rajamani, S., Sayre, R. T., Brokstein, P., Dubchak, I., Goodstein, D., Hornick, L., Huang, Y. W., Jhaveri, J., Luo, Y., Martínez, D., Ngau, W. C. A., Otillar, B., Poliakov, A., Porter, A., Szajkowski, L., Werner, G., Zhou, K., Grigoriev, I. V., Rokhsar, D. S. \& Grossman, A. R. 2007. The Chlamydomonas genome reveals the evolution of key animal and plant functions. Science 318:245-250.

Moellering, E. R. \& Benning, C. 2010. RNA interference silencing of a major lipid droplet protein affects lipid droplet size in Chlamydomonas reinhardtii. Eukaryot. Cell 9:97-106.

Ochman, H., Gerber, A. S. \& Hartl, D. L. 1988. Genetic applications of an inverse polymerase chain reaction. Genetics 120:621-623.

Polle, J. E. W., Kanakagiri, S. D. \& Melis, A. 2003. tla1, a DNA insertional transformant of the green alga Chlamydomonas reinhardtii with a truncated light-harvesting chlorophyll antenna size. Planta 217:49-59.

Pollock, S. V., Colombo, S. L., Prout, D. L. Jr., Godfrey, A. C. \& Moroney, J. V. 2003. Rubisco activase is required for optimal photosynthesis in the green alga Chlamydomonas reinhardtii in a low- $\mathrm{CO}_{2}$ atmosphere. Plant Physiol. 133:1854-1861.
Pollock, S. V., Pootakham, W., Shibagaki, N., Moseley, J. L. \& Grossman, A. R. 2005. Insights into the acclimation of Chlamydomonas reinhardtii to sulfur deprivation. Photosynth. Res. 86:475-489.

Sartory, D. P. \& Grobbelaar, J. U. 1984. Extraction of chlorophyll $a$ from freshwater phytoplankton for spectrophotometric analysis. Hydrobiologia 114:177-187.

Shimogawara, K., Fujiwara, S., Grossman, A. \& Usuda, H. 1998. High-efficiency transformation of Chlamydomonas reinhardtii by electroporation. Genetics 148:18211828.

Sizova, I., Fuhrmann, M. \& Hegemann, P. 2001. A Streptomyces rimosus aphVIII gene coding for a new type phosphotransferase provides stable antibiotic resistance to Chlamydomonas reinhardtii. Genetics 277:221-229.

Sueoka, N. 1960. Mitotic replication of deoxyribonucleic acid in Chlamydomonas reinhardi. Proc. Natl. Acad. Sci. U. S. A. 46:83-91.

Tam, L. W. \& Lefebvre, P. A. 1993. Cloning of flagellar genes in Chlamydomonas reinhardtii by DNA insertional mutagenesis. Genetics 135:375-384.

Trapnell, C., Pachter, L. \& Salzberg, S. L. 2009. TopHat: discovering splice junctions with RNA-Seq. Bioinformatics 25:1105-1111.

Wang, Z. T., Ullrich, N., Joo, S., Waffenschmidt, S. \& Goodenough, U. 2009. Algal lipid bodies: stress induction, purification, and biochemical characterization in wildtype and starchless Chlamydomonas reinhardtii. Eukaryot. Cell 8:1856-1868.

Work, V. H., Radakovits, R., Jinkerson, R. E., Meuser, J. E., Elliott, L. G., Vinyard, D. J., Laurens, L. M. L., Dismukes, G. C. \& Posewitz, M. C. 2010. Increased lipid accumulation in the Chlamydomonas reinhardtii sta7-10 starchless isoamylase mutant and increased carbohydrate synthesis in complemented strains. Eukaryot. Cell 9:12511261.

Yoshioka, S., Taniguchi, F., Miura, K., Inoue, T., Yamano, T. \& Fukuzawa, H. 2004. The novel Myb transcription factor LCR1 regulates the $\mathrm{CO}_{2}$-responsive gene $\mathrm{Cah} 1$, encoding a periplasmic carbonic anhydrase in Chlamydomonas reinhardtii. Plant Cell 16:1466-1477. 